\title{
Predictive value of platelet to lymphocyte ratio and neutrophil to lymphocyte ratio in evaluating both lung involvement and severity of patients with coronavirus disease 2019
}

Yusuf Aksu, MD, Ali U. Uslu, MD, Gülten Tarhan, PhD, NR, Mustafa Karagülle, MD.

\begin{abstract}

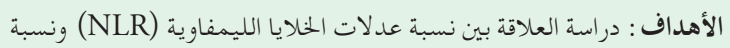

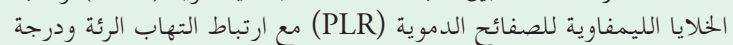

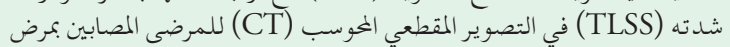

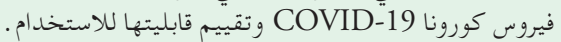

المنهجية: حصلنا على البيانات الأساسية للمختبر والخصائص السريرية

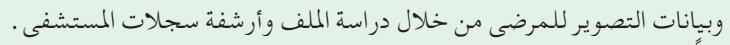

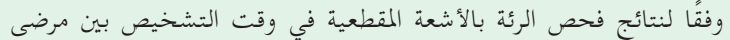
COVID-19 تم تكوين مجموعتين.

النتائج: كانت نسبة عدلات الخلايا الليمفاوية 387.11.15 2.22

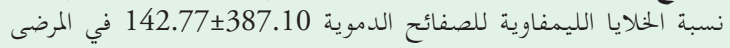

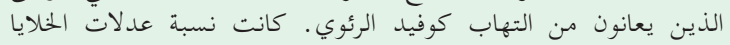

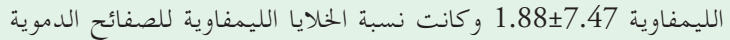

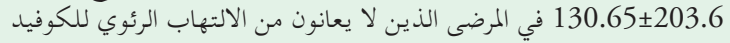

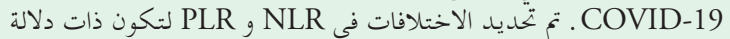

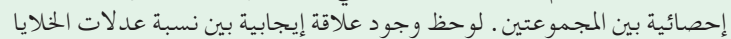

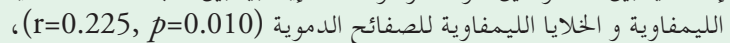
ودرجة شدة الالتهاب (r=0.244,

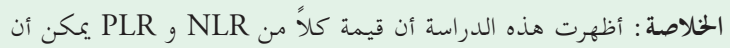

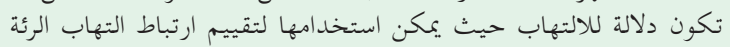

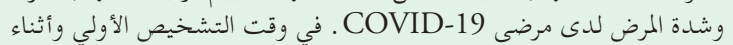

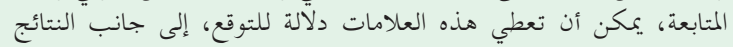

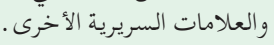

Objectives: To investigate the relationship of the neutrophil lymphocyte ratio (NLR) and the platelet lymphocyte ratio (PLR) with lung involvement and total lung severity score (TLSS) in computed tomography (CT) of patients with coronavirus disease -19 (COVID-19) and to evaluate their clinical usability.

Methods: Basic laboratory, clinical features and imaging data of patients was obtained by examining the file and archive records of our hospital. According to the findings of lung CT scan at the time of diagnosis among COVID-19 patients, 2 groups were formed.
Results: The NLR was $2.22 \pm 11.15$ and the PLR was $142.77 \pm 387.10$ in patients with COVID-19 pneumonia. The NLR was $1.88 \pm 7.47$ and the PLR was $130.65 \pm 203.68$ in patients without COVID-19 pneumonia. The differences in the NLR and the PLR were determined to be statistically significant between the 2 groups. A positive correlation was observed between NLR and PLR $(\mathrm{r}=0.225, p=0.010)$ and TLSS $(\mathrm{r}=0.244, p=0.005)$.

Conclusion: This study showed that the NLR and PLR values can be 2 inflammatory markers that can be used to evaluate lung involvement and disease severity in COVID-19 patients. At the time of initial diagnosis and during follow-up, these markers can give an idea in terms of prognosis, together with other clinical findings and markers.

Keywords: NLR, PLR, total lung severity score, COVID-19 pneumonia

Saudi Med J 2021; Vol. 42 (11): 1223-1228 doi: 10.15537/smj.2021.42.11.20210485

From the Department of Radiology (Aksu); and from the Department of Internal Medicine (Uslu, Tarhan, Karagülle), Yunus Emre State Hospital, Eskişehir, Turkey.

Received 16th June 2021. Accepted 30th September 2021.

Address correspondence and reprint request to: Dr. Yusuf Aksu, Department of Radiology, Yunus Emre State Hospital, Eskişehir, Turkey.E-mail: dryusufaksu@hotmail.com

ORCID ID: https://orcid.org/0000-0002-1696-001X

Disclosure. Authors have no conflict of interests, and the work was not supported or funded by any drug company. 
$\mathrm{P}$ athogenic viruses in the coronavirus family have caused major epidemics in the past 20 years. ${ }^{1-3}$ Coronavirus disease -19 (COVID-19) was identified as a severe acute respiratory syndrome coronavirus -2 (SARS-CoV-2); the World Health Organization (WHO) has accepted COVID-19 to be a pandemic. ${ }^{4-7}$ This disease is classified based on the severity of its clinical symptoms. While COVID-19 generally progresses with asymptomatic or mild-to-moderate symptoms, sometimes it can result in respiratory failure, multiple organ disfunction, or even death. ${ }^{8}$ Thorax computed tomography (CT) scan, which is used to diagnose the disease and determine the severity of lung involvement, plays a key role in clinical management. While the basal lower lobe of the lung is shown to be affected in CT in the literature, the most frequent patterns seen in a thorax CT are ground glass opacities, paving stones, consolidation, inter lobuler septal thickening, a pleural band, and a reticular pattern. ${ }^{\text {? }}$

There is a large number of studies on thorax CT focusing on the lung lobes and segments; these are used to evaluate the prognosis and clinical course of the disease by creating a total lung severity score (TLSS). ${ }^{10-13}$ Moreover, indicators, for instance, C-reactive protein (CRP), erythrocyte sedimentation rate (ESR), and leukocyte, ferritin, and D-dimer levels, are studied in the laboratory to evaluate the period during which the infection is active. In recent studies, these markers have been shown to be related to the course of the disease and its prognosis. Throughout the duration of the disease, neutrophilia, lymphopenia, and variable platelet levels can be seen. ${ }^{14}$ Neutrophils and platelets, which are among the immune system components found in the complete blood count, have a key role in host defense. The active cells cause the secretion of cytokines, which lead to the activation of these cells and other defense system cells. In this process, apoptosis of lymphocytes results in lymphopenia. ${ }^{15-17}$ As a result of this vicious cycle, the number of neutrophils, platelets, and lymphocytes changes, as do their ratios to each other. Lately, the neutrophil lymphocyte ratio (NLR) and the platelet lymphocyte ratio (PLR) have evolved into the most frequently used markers in infectious diseases. In some studies, these markers were reported to be related to the intensity of the COVID-19. ${ }^{18-22}$

Computed tomography is expensive and includes $\mathrm{x}$-rays. In this study, we aimed to evaluate the use of cheaper and more effective markers, NLR and PLR, in detecting lung involvement and severity in COVID-19 patients, and their usability in routine clinical workflow by comparing them with CT findings.

Methods. Our study used real-time polymerase chain reaction (RT-PCR) to evaluate the nose and throat samples of 215 patients that were determined with COVID-19 between March 10, 2020 and June 1, 2020. This study was consistent with Helsinki Declaration. After taking the Instutional Reviewer Board approval from our local ethical committee; basic laboratory, clinical features, and imaging data of the patients were obtained by examining the files and archive records of Yunus Emre State Hospital, Eskişehir, Turkey.

The patient groups who was under the age of 18 , had any systemic disease and did not have the lung CT scan, was not included in the evaluation. Patients who were found to have developed super infection during clinical follow-up were excluded from the evaluation as they may cause errors in the statistical evaluation. We included only COVID-19 patients in our study to determine whether NLR and PLR could be a marker to detect lung involvement and severity of COVID-19.

According to CT scan findings at the time of initial diagnosis, 2 groups were constructed. Group 1 consisted of cases with 131 COVID-19 pneumonia on CT findings. Group 2 consisted of 84 cases without COVID-19 pneumonia in their CT findings. Total lung severity score was obtained in group one from the data reported in the literature. The TLSS showed involvement ranging from 0-4. Lung involvement patterns of score: 1 (0-25\%), score: 2 (25-50\%), score: 3 (50-75\%), and Score: 4 (75-100\%) were accepted as involvement of each lobe. The total score was $20 .{ }^{13}$

In group one, the TLSS was calculated by evaluating the involvement levels of cases with COVID-19 pneumonia. Furthermore, the lung involvement patterns and distribution of the affected lobes were determined in the group. In the laboratory data, complete blood count, CRP, ESR, ferritin, and D-dimer levels were examined and NLR and PLR were calculated. Finally, the laboratory and imaging findings were compared between the 2 groups.

Thorax CT scan was implemented on a 16-detector CT scanner (Emotion; SIEMENS). All patients were scanned in trendelenburg position. Computed tomography images were then collected throughout a single inspiratory breath-hold time. The examining array was from the top of lung to first lomber vertebral body level. Thorax CT scan parameters: $x$-ray tube parameters $120 \mathrm{KVp}, 350 \mathrm{mAs}$; rotation time of -0.5 second; pitch -1.0 ; section thickness $-5 \mathrm{~mm}$; intersection space $-5 \mathrm{~mm}$; supplementary reformation with sharp coil kernel and a slice thickness of $1.5 \mathrm{~mm}$

The complete blood count have been implemented in the equal tester, Mindray BC-6200, which is regularly controlled each month in the chief laboratory of our hospital. Standard tubes with stable amount of 
ethylenediamine-tetraacetic acid have been used. The white blood cell, neutrophil, and lymphocyte counts were archived and the NLR was measured from these markers.

Statistical analysis. The analysis of our study was performed using the Statistical Package for Social Sciences (IBM Corp., Armonk, NY, USA). Results were presented as mean \pm standard deviation (SD) or median (minimum - maximum), mean while categorical variables were indicated as number (n) and percent (\%). Homogeneous distribution of the groups was assessed using Kolmogorov-Smirnov test. Homogeneous distribution parameters were evaluated using the Student t-test, one-sided ANOVA test in independent groups, Tukey honestly significant difference (HSD) test was used in the inter-group post-hoc evaluation, non-homogeneous distribution parameters were evaluated using the Mann-Whitney U-test, while categorical variables were reviewed by Chi-square test. The Spearman's correlation test was used for correlation analyses. The comparison of sensitivity and specificity was evaluated by receiver operating characteristic (ROC) curve graphics. Stepwise linear regression was carried out with the dependent variable TLSS and independent variables NLR, PLR and the others parameters. A $p$-value $<0.05$ was considered significant.

Results. The median age of the COVID-19 cases was $44.6 \pm 16.0$ (range: $18-83$ ); $102(47.4 \%)$ of the patients were women and $113(52.6 \%)$ were men. In group one, lobar distribution and lung involvement patterns was presented in Table 1. Lung involvement was detected in the CT of 131 (60.9\%) patients; lung involvement was not detected in the CT of 84 (39.1\%) patients. This finding was correlated with the results reported in the recent literature. The most prominent lung involvement pattern was ground glass opacity.

The age and gender characteristics of the cases with and without COVID-19 pneumonia are summarized in Table 2. The NLR was $2.22 \pm 11.15$ and the PLR was $142.77 \pm 387.10$ in group 1 . The NLR was $1.88 \pm 7.47$ and the PLR was $130.65 \pm 203.68$ in group 2. The differences in the NLR and the PLR were determined to be statistically significant among the 2 groups (Table 2).

The NLR, PLR, and other parameters are shown in Table 3, a positive relation was demonstrated between $\operatorname{NLR}(\mathrm{r}=0.225, p=0.010)$ and PLR ( $\mathrm{r}=0.244, p=0.005)$ and TLSS.

Among the COVID-19 patients, NLR showed lung involvement by ROC curve analysis, with a cut-off value of 1.87 , a sensitivity of $62 \%$, a specificity of $52 \%$, and $p=0.006$ (area under the curve [AUC]: $0.611,95 \%$
Table 1 - The baseline imaging characteristics of the COVID-19 patients with CT findings $(\mathrm{n}=131)$.

\begin{tabular}{lc}
\hline Characteristics & $\mathbf{n}(\%)$ \\
\hline Left upper lobe & $53(40.5)$ \\
Left lower lobe & $108(82.4)$ \\
Right upper lobe & $74(56.5)$ \\
Right middle lobe & $64(48.9)$ \\
Right lower lobe & $101(77.1)$ \\
Ground glass opasities & $125(95.4)$ \\
Consolidation & $65(49.6)$ \\
Reticular pattern & $78(59.5)$ \\
Crazy paving pattern & $45(34.4)$ \\
Lymphadenopathy & $12(9.2)$ \\
Pleural efussion & $5(3.8)$ \\
Total lung severity score, median (min-max) & $6.9(1-18)$ \\
\hline \multicolumn{2}{c}{ COVID-19: coronavirus disease -19, CT: computed tomography }
\end{tabular}

confidence interval [CI]: [0.534-0.687]). Platelet to lymphocyte ratio had a cut-off value of 125.47 with a sensitivity of $64 \%$, a specificity of $52 \%$, and $p=0.030$ (AUC: 0.588, 95\% CI: [0.511-0.664]) (Figure 1).

Stepwise regression analyses showed that there was an independent relationship between NLR $(\beta=4.370$, $p=0.036)$, PLR $(\beta=-4.161, p=0.024)$, and TLSS. When the relationship of NLR and PLR to TLSS was evaluated using linear regression analysis, it was seen that NLR $(\beta=4.370, p=0.036)$ and PLR $(\beta=-4.161$, $p=0.024)$ predicted TLSS independently of various variables (Table 4).

Discussion. In this study, we evaluated the relationship between NLR and PLR in COVID-19 patients and lung involvement and disease severity using CT scans. Our findings showed that both NLR and PLR were higher in COVID-19 patients with lung involvement in comparison to those without lung involvement. Moreover, we found a relationship between NLR and PLR and TLSS in patients with lung involvement on CT.

Although the RT-PCR test is the gold standard for the diagnosis of COVID-19 patients, lung CT can be used to support the diagnosis and treatment follow-up in these patients. Furthermore, it is known that the evaluation of the lung in CT may show abnormalities before the RT-PCR test. ${ }^{23}$ Xiang et al, ${ }^{24}$ reported that the CT scans in COVID-19 patients could enable the monitoring of dynamic changes in lung involvement to evaluate the density, stage, and severity of pulmonary lesions. Furthermore, it was determined that idea scoring system could be used to assess the clinical course and severity of the disease by considering the lobe and 
PLR and NLR in COVID-19 patients ... Aksu et al

Table 2 - Comparison of laboratory features of COVID-19 patients with and without CT findings.

\begin{tabular}{|c|c|c|c|}
\hline \multirow[t]{3}{*}{ Variables } & \multicolumn{2}{|c|}{ COVID-19 patients } & \multirow[t]{2}{*}{$P$-value } \\
\hline & With CT findings $(\mathrm{n}=131)$ & Without CT findings $(n=84)$ & \\
\hline & \multicolumn{2}{|c|}{ Mean $\pm S D$} & \\
\hline Age, years & $48.3 \pm 15.6$ & $38.9 \pm 15.0$ & $<0.0001$ \\
\hline \multicolumn{4}{|l|}{ Gender, n (\%) } \\
\hline Male & $58(44.3)$ & $44(52.4)$ & 0.247 \\
\hline Female & $73(55.7)$ & $40(47.6)$ & \\
\hline Hemoglobin, g/dL & $14.06 \pm 1.80$ & $14.45 \pm 1.78$ & 0.033 \\
\hline INR & $1.08 \pm 0.17$ & $1.07 \pm 0.10$ & 0.754 \\
\hline CRP, mg/L, median (min-max) & $7.95(0.60-552.33)$ & $3.36(0.60-40.60)$ & 0.010 \\
\hline Ferritin, median (min-max) & $89.30(9.60-851)$ & $85.10(7.80-776.50)$ & 0.140 \\
\hline D-dimer, ng/mL, median (min-max) & $127(18-3524)$ & $90(7-2164)$ & 0.355 \\
\hline Platelet, $\times 10^{9} / \mathrm{L}$ & $218.97 \pm 63.61$ & $221 \pm 58.28$ & 0.811 \\
\hline Leucocyte, $\times 10^{9} / \mathrm{L}$ & $5.97 \pm 2.41$ & $5.77 \pm 1.87$ & 0.508 \\
\hline Neutrophil, $\times 10^{9} / \mathrm{L}$ & $3.94 \pm 2.41$ & $3.44 \pm 1.67$ & 0.079 \\
\hline Lymphocyte, $\times 10^{9} / \mathrm{L}$ & $1.52 \pm 0.70$ & $1.71 \pm 0.59$ & 0.033 \\
\hline NLR, median (min-max) & $2.22(0.63-25.57)$ & $1.88(0.53-8.61)$ & 0.001 \\
\hline PLR, median (min-max) & $142.77(57.94-495.83)$ & $130.65(39.39-325.74)$ & 0.005 \\
\hline
\end{tabular}

COVID-19: coronavirus disease -19, CT: computed tomography, INR: international normalized ratio, CRP: C-reactive protein, NLR: neutrophil to lymphocyte ratio, PLR: platelet to lymphocyte ratio, SD: standard deviation

Table 3 - The correlation between CRP, ferritin and the other markers according to TLSS in patients with COVID-19.

\begin{tabular}{lcc}
\hline TLSS & $\mathbf{r}$ & $P$-value \\
\hline Hemoglobin, g/dL & 0.153 & 0.081 \\
CRP, mg/L & 0.599 & $<0.0001$ \\
Ferritin & 0.328 & 0.008 \\
Platelet, $\times 10^{9} / \mathrm{L}$ & -0.174 & 0.047 \\
Leucocyte, $\times 10^{9} / \mathrm{L}$ & -0.103 & 0.241 \\
Neutrophil, $\times 10^{9} / \mathrm{L}$ & 0.027 & 0.756 \\
Lymphocyte, $\times 10^{9} / \mathrm{L}$ & -0.329 & $<0.0001$ \\
D-dimer, ng $/ \mathrm{mL}$ & 0.285 & 0.013 \\
NLR & 0.225 & 0.010 \\
PLR & 0.244 & 0.005 \\
\hline
\end{tabular}

CRP: C-reactive protein, COVID-19: coronavirus disease -19, TLSS: total lung severity score, NLR: neutrophil to lymphcyte ratio, PLR: platelet to lymphocyte ratio

segment involvement in the lung using CT. Li et al, ${ }^{25}$ evaluated the relationship between the severity of the disease and the TSS performed by evaluating the lung involvement of over 5 lobes on CT. They found that the TSS was high in severe and critical cases. Zhang et al, ${ }^{26}$ scored lungs topographically by evaluating the lung at 5 levels. They showed that thorax CT scoring can predict systemic involvement and prognosis. Yang $\mathrm{R}$ et al, ${ }^{27}$ reported that, in COVID-19 patients, the severity of pulmonary involvement can be evaluated quickly and objectively using the CT severity score. Zhang et al, ${ }^{28}$ showed that the severity of lung infiltrations detected

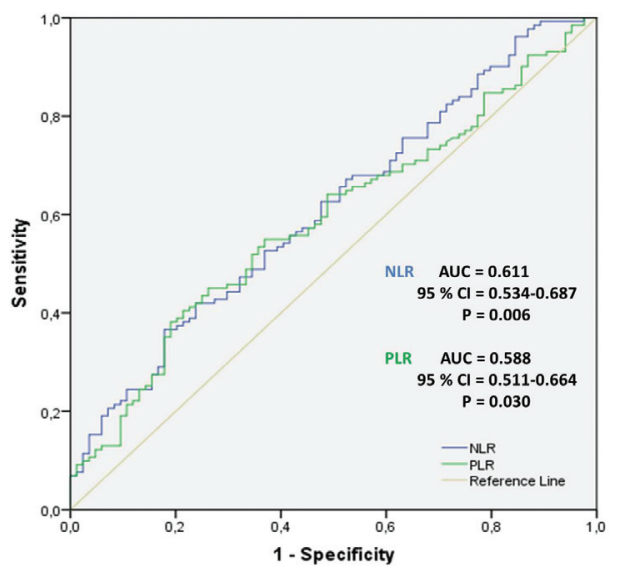

Figure 1 - Receiver-operating characteristic curve of neutrophil lymphocyte ratio (NLR) and platelet lymphocyte ratio (PLR) for predicting patients with lung involvement of coronavirus disease -19 (COVID-19) in in the study population. AUC: area under the curve, $\mathrm{CI}$ : confidence interval

in lung CT scans might be related to laboratory parameters in the early and later stages of the disease. In the same study, they evaluated COVID-19 patients radiologically in 4 stages (the early, progressive, peak, and absorption stages). They found that there were statistically significant differences in lymphocyte values in the neutrophils and thrombocytes at these stages; they also reported that there is a correlation between neutrophil counts and TLSS in the early and advanced stages of COVID-19. 
Table 4 - Linear regression analysis for TLSS in patients with coronavirus disease -19 (COVID-19).

\begin{tabular}{lcc}
\hline Independent variables & Beta regression coefficient & $P$-value \\
\hline BUN & -0.058 & 0.300 \\
Creatinine & 0.044 & 0.552 \\
ALT & -0190 & 0.243 \\
AST & 0.395 & 0.073 \\
LDH & 0.895 & 0.035 \\
INR & 0.182 & 0.151 \\
CRP & 1.839 & 0.019 \\
Ferritin & -1.738 & 0.019 \\
Platelet & -2.999 & 0.041 \\
Leucocyte & -0.841 & 0.454 \\
Neutrophil & 4.077 & 0.084 \\
Lymphocyte & -0.380 & 0.379 \\
NLR & 4.370 & 0.036 \\
PLR & -4.161 & 0.024 \\
\hline
\end{tabular}

TLSS: total lung severity score, BUN: blood urea nitrogen, ALT: alanine transaminase, AST: aspartate transaminase, $\mathrm{LDH}$ : lactate dehydrogenase,

INR: international normalized ratio, CRP: C-reactive protein, NLR: neutrophil to lymphocyte ratio, PLR: platelet to lymphocyte ratio

More than $50 \%$ of the bone marrow is responsible for the production of neutrophils. These cells are the first line of defense in the immune system. Neutrophils play an active role in the production of many lyticenzymes, free oxygen radicals, and cytokines. The role of cytokines is very important in the pathogenesis of many infectious diseases, especially COVID-19..$^{15-17}$ Neutrophils and platelets are involved in the production of cytokines, and cytokines also contribute to the activation of neutrophils and platelets. Studies have shown that platelets play an active role in inflammation and they have regulatory properties on the immune system. ${ }^{29}$ After this complex process occurs, changes in lymphocyte levels can be observed. In COVID-19 patients, it is thought that lymphopenia may develop due to the attack of lymphocytes expressing the angiotensinconverting enzyme receptor by SARS-CoV-2, with the apoptosis of lymphocytes that increase secondary to the inflammatory process and the released cytokines. Moreover, lymphocyte proliferation defects can be observed as a result of metabolic disorders, such as the reticulo endothelial system (such as the spleen and thymus) and possible hyperlactic acidemia. ${ }^{18-20}$ When neutrophils, platelets, and lymphocytes are evaluated in COVID-19 patients, it is predicted that inflammatory markers, such as NLR and PLR, which are composed of these components, may cause some changes in this disease.

$\mathrm{Qu}$ et al, ${ }^{21}$ found that the increased PLR values in COVID-19 patients were associated with the clinical course of the disease and the length of hospital stay. In the same study, they reported that the cytokine storm, which is among the most important complications of COVID-19, affected the platelet and PLR levels. Yang et $a l,{ }^{22}$ found that NLR may be related to the severity of the infection and that a high NLR is an independent prognostic biomarker for COVID-19 patients. In their semi-quantitative segmental CT scoring study, Yang et $\mathrm{al}^{22}$ compared the initial thoracic tomography of the patients. They confirmed that the thorax CT score was higher in COVID-19 patients than non-severe patients and that the first CT scan could be used in the triaj of the patients. ${ }^{27}$ Chan et al, ${ }^{29}$ evaluated a total of 20 studies in their meta-analysis and reported that NLR and PLR can be used as independent prognostic markers of disease severity in COVID-19.

When neutrophils, thrombocytes, and lymphocytes are evaluated in COVID-19 patients, it is predicted that inflammatory markers, such as NLR and PLR, which consist of these components, may result in some changes in this disease.

In our study, NLR and PLR results were higher in patients with lung involvement in comparison to those without lung involvement. This was correlated with the TLSS, the evaluation of lung findings in CT with ROC analysis, and linear regression analysis, suggesting that NLR and PLR may be markers that can be used in the follow-up of disease severity in COVID-19 patients.

Study limitations. This is a retrospective study and the data is based on the CT findings and laboratory values of the patients at the time of initial diagnosis. Moreover, the effects of the control CT findings and the treatments applied on the bone marrow were not evaluated.

In conclusion, there are many studies related to lung involvement and severity of COVID-19; some of them claimed that thoracic CT showed lung involvement and severity of COVID-19, others claimed that PLR and NLR showed lung involvement and severity of COVID-19. This study verified that NLR and PLR values can be 2 inflammatory indicators that can be used to demonstrate lung involvement and disease severity in COVID-19 patients. With these results, NLR and PLR can be among the indicators that can be used to figure out disease severity in COVID-19 patients when supported by different prospective studies.

Acknowledgment. The authors gratefully acknowledge Scribendi (www.scribendi.com) for English language editing.

\section{References}

1. Petrosillo N, Viceconte G, Ergonul O, Ippolito G, Petersen E. COVID-19, SARS and MERS: are they closely related? Clin Microbiol Infect 2020; 26: 729-734. 
2. Prompetchara E, Ketloy C, Palaga T. Immune responses in COVID-19 and potential vaccines: lessons learned from SARS and MERS epidemic. Asian Pac J Allergy Immunol 2020; 38: 1-9.

3. De Wit E, van Doremalen N, Falzarano D, Munster VJ. SARS and MERS: recent insights into emerging coronaviruses. Nat Rev Microbiol 2016; 14: 523-534.

4. World Health Organization. General's remarks at the media briefing on 2019-nCoV on 11 February 2020. [Updated 2020; accessed 2020 February 11]. Available from: https://www.who. int $/ \mathrm{dg} / \% 20$ speeches/detail/who-director-general-s-remarks-atthe-media-briefingon-2019-ncov-on-11-february-2020

5. Li Q, Guan X, Wu P, Wang X, Zhou L, Tong Y, et al. Early transmission dynamics in Wuhan, China, of novel coronavirusinfected pneumonia. N Engl J Med 2020; 382: 1199-1207.

6. Carlos WG, Dela Cruz CS, Cao B, Pasnick S, Jamil S. Novel Wuhan (2019-nCoV) coronavirus. Am J Respir Crit Care Med 2020; 201: P7-P8.

7. Zhu J, Ji P, Pang J, Zhong Z, Li H, He C, et al. Clinical characteristics of 3062 COVID-19 patients: a meta-analysis. $J$ Med Virol 2020; 92: 1902-1914.

8. Zhu T, Wang Y, Zhou S, Zhang N, Xia L. A comparative study of chest computed tomography features in young and older adults with corona virus disease (COVID-19). J Thorac Imaging 2020; 35: W97-W101.

9. Francone M, Iafrate F, Masci GM, Coco S, Cilia F, Manganaro L, et al. Chest CT score in COVID-19 patients: correlation with disease severity and short-term prognosis. Eur Radiol 2020; 30: 6808-6817.

10. Xie X, Zhong Z, Zhao W, Zheng C, Wang F, Liu J. Chest CT for typical coronavirus disease 2019 (COVID-19) pneumonia: relationship to negative RT-PCR testing. Radiology 2020; 296: E41-E45.

11. Pan F, Ye T, Sun P, Gui S, Liang B, Li L, et al. Time course of lung changes at chest CT during recovery from coronavirus disease 2019 (COVID-19). Radiology 2020; 295: 715-721.

12. Bernheim A, Mei X, Huang M, Yang Y, Fayad ZA, Zhang N, et al. Chest CT findings in coronavirus disease-19 (COVID-19): relationship to duration of infection. Radiology 2020; 295: 200463.

13. Zhang J, Meng G, Li W, Shi B, Dong H, Su Z, et al. Relationship of chest CT score with clinical characteristics of 108 patients hospitalized with COVID-19 in Wuhan, China. Respir Res 2020; 21: 180.

14. Zhu N, Zhang D, Wang W, Li X, Yang B, Song J, et al. A novel coronavirus from patients with pneumonia in China, 2019. N Engl J Med 2020; 382: 727-733.

15. Heeb LEM, Egholm C, Impellizzieri D, Ridder F, Boyman O. Regulation of neutrophils in type 2 immune responses. Curr Opin Immunol 2018; 54: 115-122.
16. Hvas AM, Grove EL. Platelet function tests: preanalytical variables, clinical utility, advantages, and disadvantages. Methods Mol Biol 2017; 1646: 305-320.

17. Xu H, Zhong L, Deng J, Peng J, Dan H, Zeng X, et al. High expression of ACE2 receptor of 2019-nCoV on the epithelial cells of oral mucosa. Int J Oral Sci 2020; 12: 8.

18. Huyut MT, İlkbahar F. The effectiveness of blood routine parameters and some biomarkers as a potential diagnostic tool in the diagnosis and prognosis of Covid-19 disease. Int Immunopharmacol 2021; 98: 107838.

19. Harmon C, O'Farrelly C, Robinson MW. The immune consequences of lactate in the tumor microenvironment. $A d v$ Exp Med Biol 2020; 1259: 113-124.

20. Zhong Z, Huang Y, Liu Y, Chen J, Liu M, Huang Q, et al. Correlation between $\mathrm{C}$-reactive protein to albumin ratio and disease activity in patients with axial spondyloarthritis. Dis Markers 2021; 2021: 6642486.

21. Qu R, Ling Y, Zhang YH, Wei LY, Chen X, Li XM, et al. Platelet-to-lymphocyte ratio is associated with prognosis in patients with coronavirus disease-19. J Med Virol 2020; 92: 1533-1541.

22. Yang AP, Liu JP, Tao WQ, Li HM. The diagnostic and predictive role of NLR, d-NLR and PLR in COVID-19 patients. Int Immunopharmacol 2020; 84: 106504.

23. Pan Y, Guan H, Zhou S, Wang Y, Li Q, Zhu T, et al. Initial CT findings and temporal changes in patients with the novel coronavirus pneumonia (2019-nCoV): a study of 63 patients in Wuhan, China. Eur Radiol 2020; 30: 3306-3309.

24. Xiang Y, Yang Q, Sun H, Qin X, Li X, Zhang Q. [Chest CT findings and their dynamic changes in patients with COVID-19]. Nan Fang Yi Ke Da Xue Xue Bao 2020; 40: 327-332. [in Chinese]

25. Li K, Fang Y, Li W, Pan C, Qin P, Zhong Y, et al. CT image visual quantitative evaluation and clinical classification of coronavirus disease (COVID-19). Eur Radiol 2020; 30: 4407-4416.

26. Zhang MQ, Wang XH, Chen YL, Zhao KL, Cai YQ, An CL, et al. [Clinical features of 2019 novel coronavirus pneumonia in the early stage from a fever clinic in Beijing]. Zhonghua Jie He He Hu Xi Za Zhi 2020; 43: E013. [in Chinese]

27. Yang R, Li X, Liu H, Zhen Y, Zhang X, Xiong Q, et al. Chest CT severity score: an imaging tool for assessing severe COVID-19. Radiol Cardiothorac Imaging 2020; 2: e200047.

28. Zhang B, Zhang J, Chen H, Chen L, Chen Q, Li M, et al. Novel coronavirus disease 2019 (COVID-19): relationship between chest CT scores and laboratory parameters. Eur J Nucl Med Mol Imaging 2020; 47: 2083-2089.

29. Chan AS, Rout A. Use of neutrophil-to-lymphocyte and platelet-to-lymphocyte ratios in COVID-19. J Clin Med Res 2020; 12: 448-453. 